\title{
Pre-radiotherapy neutrophil-to-lymphocyte ratio as an independent prognostic factor in patients with locally advanced hepatocellular carcinoma treated with radiotherapy
}

\author{
Seok Hyun Son ${ }^{1}$, Eun Young Park ${ }^{1}$, Hee Hyun Park ${ }^{1}$, Chul Seung Kay ${ }^{1}$, Hong Seok \\ Jang ${ }^{2}$ \\ ${ }^{1}$ Department of Radiation Oncology, Incheon St. Mary's Hospital, College of Medicine, The Catholic University of Korea, \\ Seoul, Korea \\ ${ }^{2}$ Department of Radiation Oncology, Seoul St. Mary's Hospital, College of Medicine, The Catholic University of Korea, Seoul, \\ Korea
}

Correspondence to: Seok Hyun Son, email: sonshyun@catholic.ac.kr

Keywords: neutrophil-to-lymphocyte ratio, radiotherapy, hepatocellular carcinoma

Received: November 01, $2016 \quad$ Accepted: January 27, $2017 \quad$ Published: February 09, 2017

\section{ABSTRACT}

We aimed to investigate the pre-radiotherapy neutrophil-to-lymphocyte ratio (prNLR) as a prognostic factor in patients with locally advanced hepatocellular carcinoma (HCC) treated with radiotherapy (RT), and to determine the optimal cut-off value for prNLR. We retrospectively evaluated 56 patients with locally advanced HCC treated with RT (helical tomotherapy) between March 2006 and February 2012. The optimal cut-off value was determined by using a maximally selected log-rank test. Prognostic factors that influence the local progression-free survival (PFS) and overall survival (OS) were evaluated. A prNLR of 2.1 was determined to be the optimal cut-off value. In a comparison between the high-prNLR group and the low-prNLR group, there was a 13.1-month difference in the median OS (10.3 vs. 23.4 months, $p=0.003)$ and a 10.4-month difference in the median local PFS (7.1 vs. 17.5 months, $p=0.001)$. On multivariate analysis of prognostic factors for local PFS and OS, the prNLR was identified as an independent prognostic factor, and the hazard ratio was 4.2 and 2.5, respectively. We demonstrated that a low prNLR was significantly associated with better PFS and OS in patients with locally advanced HCC treated with RT, and the prNLR should be considered as an independent prognostic factor in these patients.

\section{INTRODUCTION}

The standard treatments for unresectable locally advanced hepatocellular carcinoma (HCC) are transarterial chemoembolization (TACE) and sorafenib. TACE is currently recommended for large multinodular HCC [1,2], whereas sorafenib is the suggested first-line treatment for HCC with vascular invasion or extrahepatic spread $[3,4]$. Although radiotherapy (RT) has not been a main modality in the treatment of locally advanced HCC, several studies have suggested RT as an effective treatment option for patients with locally advanced HCC [5-7]. As RT has been more increasingly used with TACE or sorafenib, the knowledge of prognostic factors associated with RT has become important in the selection of optimal patients and in designing the RT strategy.

Recently, increasing evidences has shown that the presence of systemic inflammation correlates with poorer survival in some cancer patients [8-13]. Several studies have shown that the pretreatment neutrophil-tolymphocyte ratio (NLR) was an independent prognostic factor in patients with HCC treated with surgical resection, transplantation, TACE, radiofrequency ablation (RFA), and sorafenib $[10,14-20]$. The NLR is the ratio of the neutrophil count to the lymphocyte count, and is a useful index that reflects systemic inflammatory response in cancer patients [21, 22]. However, the range of previously reported cut-off values for NLR was from about 1.0 to 5.0 , which is too wide for selecting an optimal and effective value in the real clinical setting. In addition, to our knowledge, there is no study on the prognostic role of NLR in patients with HCC treated with RT.

In this study, we aimed to investigate the preradiotherapy NLR (prNLR) as a prognostic factor in patients with locally advanced HCC treated with RT, and to determine the optimal cut-off value for the prNLR. 


\section{RESULTS}

\section{Optimal cut-off value for prNLR, and comparison between NLR groups stratified according to the cut-off value}

The optimal cut-off value for predicting prognosis was calculated by using the maximally selected log-rank test, for which prNLR levels and local PFS were used as variables. According to the results of this statistical test, a NLR of 2.1 was determined to be a significant cut-off value of the prNLR. With this cut-off value, all 56 patients were divided in two groups: low-prNLR group (prNLR $<2.1$ ) and highprNLR group (prNLR $\geq 2.1$ ). Sixteen patients $(28.6 \%$ ) were identified as the low-prNLR group, and the remaining 40 patients (71.4\%) were identified as the high-prNLR group. The clinical characteristics were compared between the two prNLR groups, and described in Table 1. Age, sex, ECOG PS status, AJCC stage, presence or absence of hepatitis, liver cirrhosis, or PVTT, AFP levels, pretreatment CP class, and GTV were not different between the two NLR groups.

\section{Local PFS and OS}

At the time of analysis, 13 patients $(23.2 \%)$ developed actual local progression (1 patient: alive, 12 patients: deceased), 34 patients $(60.7 \%)$ were deceased without evidence of local progression, and overall, 46 patients $(82.1 \%)$ were deceased. The median follow-up duration was 13.2 months (range, 3.5-85.3 months). The median OS was 13.6 months, and the 1- and 2-year OS rates were $51.8 \%$ and $23.1 \%$, respectively. The median local PFS was 10.5 months, and the 1- and 2-year local PFS rates were $42.4 \%$ and $12.6 \%$, respectively.

\section{Clinical factors that influence the local PFS}

In univariate analysis, female sex, presence of liver cirrhosis, $\mathrm{AFP} \geq 400 \mathrm{IU} / \mathrm{mL}$, pretreatment $\mathrm{CP}$ class $\mathrm{B}$, and prNLR $\geq 2.1$ were identified to be statistically significant unfavorable factors for local PFS ( $p=0.028,0.0015,0.004$, 0.035 , and 0.001 , respectively). Age, ECOG performance status, AJCC stage, presence or absence of hepatitis or PVTT, and GTV were not found to be statistically significant factors. In multivariate analysis, female sex, presence of liver cirrhosis, AFP $\geq 400 \mathrm{IU} / \mathrm{mL}$, and prNLR $\geq 2.1$ were identified to be statistically significant unfavorable factors for local PFS ( $p=0.006,0.020,0.016$, and $<0.001$, respectively). The results of univariate and multivariate analyses are summarized in Table 2.

\section{Clinical factors that influence the OS}

In univariate analysis, age $\geq 60$ years, presence of liver cirrhosis, AFP $\geq 400 \mathrm{IU} / \mathrm{mL}$, and $\mathrm{prNLR} \geq 2.1$ were identified to be statistically significant unfavorable factors for
OS ( $p=0.035,0.010,<0.001$, and 0.004 , respectively). Sex, ECOG performance status, AJCC stage, presence or absence of hepatitis or PVTT, pretreatment CP class, and GTV were not found to be statistically significant factors. In multivariate analysis, presence of liver cirrhosis, $\mathrm{AFP} \geq 400 \mathrm{IU} / \mathrm{mL}$, and prNLR $\geq 2.1$ were identified to be statistically significant unfavorable factors for OS $(p=0.048,<0.001$, and 0.023 , respectively). The results of univariate and multivariate analyses are summarized in Table 3.

\section{Significance of prNLR in patients with locally advanced HCC treated with RT}

In the low-prNLR group, the median OS was 23.4 months, and the 1- and 2-year OS rates were $81.3 \%$, and $45.7 \%$, respectively. The median local PFS was 17.5 months, and the 1- and 2-year local PFS rates were $75.0 \%$ and $39.8 \%$, respectively (Figure 1). In the high-prNLR group, the median OS was 10.3 months, and the 1- and 2-year OS rates were $40.0 \%$ and $14.1 \%$, respectively. The median local PFS was 7.1 months, and the 1- and 2-year local PFS rates were $29.0 \%$ and $0.0 \%$, respectively (Figure 1 ). In a comparison between the high-prNLR and low-prNLR groups, there was a 13.1-month difference in the median OS (10.3 vs. 23.4 months, $p=0.003)$ and the relative risk of mortality was 2.5 . There was 10.4 -month difference in the median local PFS (7.1 vs. 17.5 months, $p=0.001$ ), and the relative risk of disease progression was 4.2.

\section{DISCUSSION}

NLR is a useful index that reflects systemic inflammatory response in some cancer patients [21, 22]. An increased neutrophil level was related to the systemic releases of chemokines and interleukins, which promote tumor growth and metastasis in HCC [23]. Lymphocytes are related to a T lymphocyte-mediated antitumor response [24], and a decreased lymphocyte number reflects a weaker lymphocyte-mediated immune response to the tumor [25]. Therefore, the NLR reflects the potential balance between neutrophil-associated pro-tumor inflammation and lymphocyte-dependent antitumor immune function; a high NLR is likely to reflect a more aggressive disease and poor prognosis [17, 26, 27].

Previous studies have investigated the value of NLR as a prognostic factor for HCC. The measurement of the NLR would be helpful in predicting the prognosis of malignant cancers such as gastric [12], lung [13], breast [8], pancreatic [11], and colorectal [9] cancers. In patients with $\mathrm{HCC}$, a prognostic role of the NLR has been investigated after treatments including surgical resection $[10,17]$, transplantation [14], RFA [16], TACE [18-20], and sorafenib [15]. In addition, a recently reported metaanalysis demonstrated the role of the NLR [26]. This analysis revealed that low baseline NLR was significantly associated with better OS (hazard ratio [HR], 1.80; 95\% confidence interval $[\mathrm{CI}], 1.59-2.04, p<0.001)$ and 
recurrence-free or disease-free survival (HR, 1.80; 95\% CI, $1.80-2.76, p<0.001)$. Another finding was that low post-treatment NLR was significantly associated with better OS (HR, 1.90; 95\% CI, 1.22-2.93, $p<0.001)$. In addition, a decreased NLR after treatment was significantly associated with better OS (HR, 2.23; 95\% CI, 1.83-2.72, $p<0.001$ ) and recurrence-free or diseasefree survival of patients with HCC (HR, 2.23; 95\% CI, $1.83-2.72, p<0.001)$.

However, to our knowledge, there is no study on the prognostic role of the NLR in patients with HCC treated with RT. Therefore, in this study, we investigated the prognostic significance of the prNLR in patients with HCC who received helical tomotherapy with a hypofractionated scheme. This study showed the significant survival benefit in the low-prNLR group, which is consistent with several previous reports. In a comparison between high-prNLR and low-prNLR group, there was a 13.1-month difference in the median OS (10.3 vs. 23.4 months, $p=0.003$ ) and a 10.4-month difference in the median local PFS ( 7.1 vs. 17.5 months, $p=0.001$ ). On multivariate analysis of prognostic factors for local PFS and OS, the prNLR was identified as an independent prognostic factor, and the HR was 4.2 and 2.5 , respectively.

To predict prognosis according to the NLR, determination of the cut-off value for the NLR is the most important point. In their study, Terashima et al. set the cutoff level of the NLR as the median value [19]. Another study determined the cut-off value by comparing the survival rates between NLR groups stratified according to several candidate values from 2.6 to 3.2 [28]. Several studies determined cut-off values by using receiver-operating characteristic (ROC) curves [17, 22]. In those studies, tumor response was selected as an endpoint to generate the ROC curve. However, those cut-off values were actually optimal for predicting tumor response, not for directly predicting survival. In this study, we chose a different statistical method to calculate the optimal cut-off value representing a direct association with local PFS. The cut-off value was calculated by using a maximally selected log-rank test, in which the presence or absence of local PFS, local PFS duration, and prNLR levels were used as variables [29]. Despite a different statistical method, the cut-off value of this study was similar to previously reported values. The range of reported cutoff values of the NLR was from about 1.0 to 5.0 , which is too wide. According to the meta-analysis by Qi et al., the statistically significant cut-off value was 5.0 or within the range from about 2.0 to 3.0 [26]. Our cut-off value of 2.1 is within this range and was also statistically significant. Considering the statistical method for determining the cutoff value, our cut-off value is considered more optimal.

$\mathrm{Li}$ et al. reported that a low level of NLR was associated with lower AFP, ALP, and total bilirubin, as well as decreased incidences of ascites, portal vein thrombosis, and metastasis, all of which reflect better prognosis [22]. According to Okamura et al., lower AFP and smaller tumor size were associated with a low NLR [28]. Although the association between the NLR and other prognostic factors could be a valid explanation for the difference in prognosis, it was still controversial. In this study, there was no difference in other prognostic factors between the low-prNLR and high-prNLR groups. This absence of association with other prognostic factors could be a good explanation for why the prNLR should be considered an independent prognostic factor.

The results of this study should be carefully interpreted due to the retrospective nature of this study and the relatively small number of cases. Although our results were obtained using proper statistical analyses, they have not been sufficiently validated for generalization. Therefore, further large-scale validation studies are needed to confirm our results.

In conclusion, we demonstrated that a low prNLR was significantly associated with better PFS and OS in patients with locally advanced HCC treated with RT. The prNLR should be considered as another independent prognostic factor in case of RT.
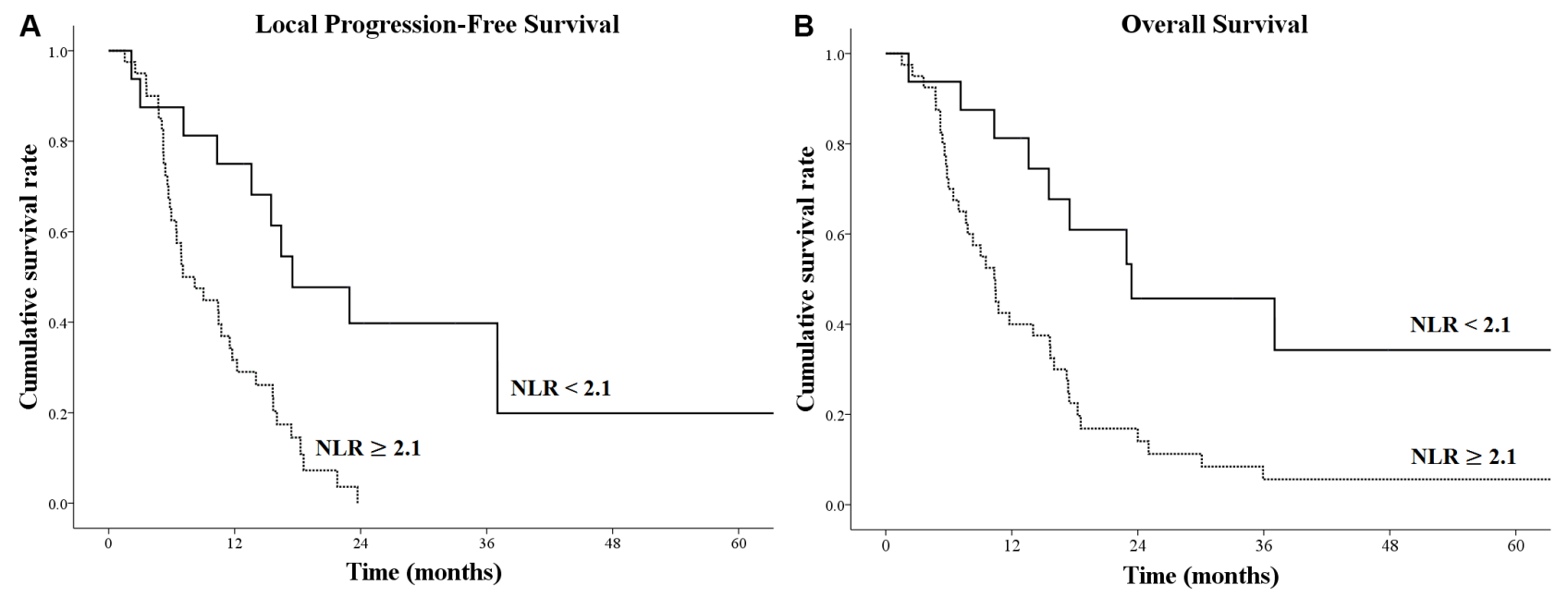

Figure 1: Kaplan-Meier survival curves for (A) local progression-free survival and (B) overall survival according to different levels of pre-radiotherapy neutrophil-to-lymphocyte ratio (prNLR). 
Table 1: Clinical characteristics associated with the level of prNLR

\begin{tabular}{|c|c|c|c|}
\hline Variables & $\operatorname{prNLR}<2.1$ & $\operatorname{prNLR} \geq 2.1$ & $p$ value \\
\hline Sex & & & 0.779 \\
\hline Male & 11 & 29 & \\
\hline Female & 5 & 14 & \\
\hline Age & & & 0.538 \\
\hline Median & 56 & 61 & \\
\hline Range & $39-80$ & $21-80$ & \\
\hline ECOG PS & & & 0.067 \\
\hline 0 & 9 & 12 & \\
\hline 1 & 7 & 28 & \\
\hline AJCC stage & & & 0.257 \\
\hline II & 4 & 4 & \\
\hline III & 10 & 33 & \\
\hline IV & 2 & 3 & \\
\hline Hepatitis & & & 0.928 \\
\hline B & 11 & 27 & \\
\hline Others & 5 & 13 & \\
\hline Liver cirrhosis & & & 0.350 \\
\hline No & 6 & 10 & \\
\hline Yes & 10 & 30 & \\
\hline PVTT & & & 0.089 \\
\hline No & 10 & 15 & \\
\hline Yes & 6 & 25 & \\
\hline AFP & & & 0.708 \\
\hline$<400$ & 12 & 28 & \\
\hline$\geq 400$ & 4 & 12 & \\
\hline Pretreatment $\mathrm{CP}$ class & & & 0.645 \\
\hline A & 14 & 33 & \\
\hline B & 2 & 7 & \\
\hline GTV & & & 0.495 \\
\hline$<214 \mathrm{~cm}^{3}$ & 11 & 31 & \\
\hline$\geq 214 \mathrm{~cm}^{3}$ & 5 & 9 & \\
\hline
\end{tabular}

Abbreviations: prNLR, pre-radiotherapy neutrophil-to-lymphocyte ratio; ECOG PS, Eastern Cooperative Oncology Group performance status; AJCC, American Joint Committee on Cancer; PVTT, portal vein tumor thrombosis; AFP, alphafetoprotein; CP, Child-Pugh; GTV = gross tumor volume.

\section{MATERIALS AND METHODS}

\section{Patients}

The inclusion criteria were as follows: 1) unresectable, locally advanced HCC; 2) age > 18 years; 3 ) a Child-Pugh
(CP) score of 5, 6, or 7 within 1 month before RT; 4) an Eastern Cooperative Oncology Group (ECOG) performance status of 0 or 1; 5) an absence of distant metastases; 6) one or more laboratory tests before, during, and after RT; 6) one or more radiological evaluations before and after RT; and 7) a prescription dose of 40-50 Gy in 10 fractions. 
Table 2: Prognostic factors that influence the local progression-free survival

\begin{tabular}{|c|c|c|c|c|}
\hline \multirow{2}{*}{ Variables } & \multicolumn{2}{|c|}{ Univariate analysis } & \multicolumn{2}{|c|}{ Multivariate analysis } \\
\hline & HR $(95 \%$ CI) & $p$ value & HR (95\% CI) & $p$ value \\
\hline Sex (Female) & $2.045(1.081-3.876)$ & 0.028 & $2.566(1.308-5.035)$ & $0.006^{*}$ \\
\hline Age $(\geq 60)$ & $1.282(0.956-1.721)$ & 0.097 & & \\
\hline ECOG PS (1) & $1.297(0.714-2.352)$ & 0.393 & & \\
\hline AJCC stage (II) & $0.678(0.196-2.343)$ & 0.539 & & \\
\hline AJCC stage (III) & $1.009(0.357-2.852)$ & 0.986 & & \\
\hline Hepatitis (B) & $1.106(0.549-1.879)$ & 0.959 & & \\
\hline Liver cirrhosis (presence) & $2.427(1.190-4.926)$ & 0.015 & $2.456(1.150-5.244)$ & $0.020^{*}$ \\
\hline PVTT (presence) & $1.742(1.355-3.154)$ & 0.067 & & \\
\hline $\operatorname{AFP}(\geq 400)$ & $2.570(1.355-4.878)$ & 0.004 & $2.281(1.164-4.470)$ & $0.016^{*}$ \\
\hline Pretreatment $\mathrm{CP}$ class $(\mathrm{B})$ & $2.487(1.064-5.813)$ & 0.035 & $2.340(0.961-5.695)$ & 0.061 \\
\hline GTV $\left(\geq 214 \mathrm{~cm}^{3}\right)$ & $1.145(0.592-2.217)$ & 0.687 & & \\
\hline Previous chemotherapy (presence) & $0.621(0.305-1.262)$ & 0.188 & & \\
\hline $\operatorname{prNLR}(\geq 2.1)$ & $3.610(1.680-7.751)$ & 0.001 & $4.211(1.930-9.188)$ & $<0.001^{\prime}$ \\
\hline
\end{tabular}

Abbreviations: HR, hazard ratio; CI, confidence interval; ECOG PS, Eastern Cooperative Oncology Group performance status; AJCC, American Joint Committee on Cancer; PVTT, portal vein tumor thrombosis; AFP, alpha-fetoprotein; CP, Child-Pugh; GTV, gross tumor volume; prNLR, pre-radiotherapy neutrophil-to-lymphocyte ratio.

"Statistically significant.

Table 3: Prognostic factors that influence the overall survival

\begin{tabular}{|c|c|c|c|c|}
\hline \multirow{2}{*}{ Variables } & \multicolumn{2}{|c|}{ Univariate analysis } & \multicolumn{2}{|c|}{ Multivariate analysis } \\
\hline & HR $(95 \%$ CI) & $p$ value & HR $(95 \%$ CI) & $p$ value \\
\hline Sex (Female) & $1.706(0.904-3.215)$ & 0.099 & & \\
\hline Age $(\geq 60)$ & $1.919(1.048-3.508)$ & 0.035 & $1.417(0.752-2.670)$ & 0.281 \\
\hline ECOG PS (1) & $1.161(0.637-2.118)$ & 0.624 & & \\
\hline AJCC stage (II) & $0.582(0.169-2.009)$ & 0.392 & & \\
\hline AJCC stage (III) & $0.720(0.253-2.047)$ & 0.537 & & \\
\hline Hepatitis (B) & $0.957(0.516-1.776)$ & 0.892 & & \\
\hline Liver cirrhosis (presence) & $2.557(1.254-5.208)$ & 0.010 & $2.065(1.006-4.240)$ & $0.048^{*}$ \\
\hline PVTT (presence) & $1.438(0.798-2.597)$ & 0.226 & & \\
\hline $\operatorname{AFP}(\geq 400)$ & $3.597(1.824-7.092)$ & $<0.001$ & $3.605(1.791-7.258)$ & $<0.001^{*}$ \\
\hline Pretreatment CP class (B) & $1.538(0.684-3.460)$ & 0.297 & & \\
\hline $\operatorname{GTV}\left(\geq 214 \mathrm{~cm}^{3}\right)$ & $0.834(0.432-1.652)$ & 0.605 & & \\
\hline Previous chemotherapy (presence) & $0.568(0.279-1.155)$ & 0.118 & & \\
\hline $\operatorname{prNLR}(\geq 2.1)$ & $2.941(1.404-6.134)$ & 0.004 & $2.474(1.133-5.402)$ & $0.023^{*}$ \\
\hline
\end{tabular}

Abbreviations: HR, hazard ratio; CI, confidence interval; ECOG PS, Eastern Cooperative Oncology Group performance status; AJCC, American Joint Committee on Cancer; PVTT, portal vein tumor thrombosis; AFP, alpha-fetoprotein; CP, Child-Pugh; GTV, gross tumor volume; prNLR, pre-radiotherapy neutrophil-to-lymphocyte ratio.

"Statistically significant.

A total of 56 patients were found to be eligible for this study. From March 2006 to February 2012, all patients received RT by using the TomoTherapy Hi-Art system (TomoTherapy Inc., Madison, WI, USA) at Incheon
St. Mary's Hospital and Seoul St. Mary's Hospital. The patients' data were retrospectively reviewed following institutional review board (IRB) approval (IRB of Incheon St. Mary's Hospital, reference no. OC16RISI0144). 
Table 4: Patients' characteristics

\begin{tabular}{|c|c|c|}
\hline Variables & $n$ & $\%$ \\
\hline \multicolumn{3}{|l|}{ Sex } \\
\hline Male & 40 & 71.4 \\
\hline Female & 16 & 28.6 \\
\hline \multicolumn{3}{|l|}{ Age (years) } \\
\hline Median & 69 & \\
\hline Range & $21-80$ & \\
\hline \multicolumn{3}{|l|}{ ECOG PS } \\
\hline 0 & 21 & 37.5 \\
\hline 1 & 35 & 62.5 \\
\hline \multicolumn{3}{|l|}{ Hepatitis } \\
\hline None & 6 & 10.7 \\
\hline $\mathrm{HBV}$ & 38 & 67.9 \\
\hline $\mathrm{HCV}$ & 5 & 8.9 \\
\hline Alcoholic & 7 & 12.5 \\
\hline \multicolumn{3}{|l|}{ Liver cirrhosis } \\
\hline No & 16 & 28.6 \\
\hline Yes & 40 & 71.4 \\
\hline \multicolumn{3}{|l|}{ PVTT } \\
\hline No & 25 & 44.6 \\
\hline Yes & 31 & 55.4 \\
\hline \multicolumn{3}{|l|}{$\operatorname{AFP}(\mathrm{IU} / \mathrm{mL})$} \\
\hline$<400$ & 40 & 71.4 \\
\hline$\geq 400$ & 16 & 28.6 \\
\hline \multicolumn{3}{|c|}{$\mathrm{CP}$ class before radiotherapy } \\
\hline A & 47 & 83.9 \\
\hline $\mathrm{B}$ & 9 & 16.1 \\
\hline \multicolumn{3}{|l|}{ AJCC stage } \\
\hline II & 8 & 14.3 \\
\hline III & 43 & 76.8 \\
\hline IVA & 5 & 8.9 \\
\hline \multicolumn{3}{|l|}{ Previous treatment } \\
\hline None & 6 & 12.5 \\
\hline TACE & 49 & 87.5 \\
\hline RFA & 7 & 12.5 \\
\hline PEI & 4 & 7.1 \\
\hline Chemotherapy & 12 & 21.4 \\
\hline
\end{tabular}

Abbreviations: ECOG PS, Eastern Cooperative Oncology Group performance status; HBV, hepatitis B virus; HCV, hepatitis C virus; PVTT, portal vein tumor thrombosis; AFP, alpha-fetoprotein; CP class, Child-Pugh class; AJCC, American Joint Committee on Cancer; TACE, transarterial chemoembolization; RFA, radiofrequency ablation; PEI, percutaneous ethanol injection.

Age, sex, ECOG performance status, American Joint Committee on Cancer (AJCC) stage (seventh edition), pretreatment CP class, absence or presence of hepatitis, liver cirrhosis, or portal vein tumor thrombosis (PVTT), alpha-fetoprotein (AFP) level, and prNLR were evaluated. Before RT, TACE was implemented in 49 patients (median number of procedures, 2; range, 1-11), percutaneous ethanol injection in 4 patients (median number of procedures, 2; range, 1-3), RFA in 7 patients (median number of procedures, 2; range, 1-3), and systemic chemotherapy in 12 patients. The patients' characteristics are described in Table 4. 


\section{Radiotherapy}

For the simulations, the patients were immobilized by using the BodyFix system (Medical Intelligence $\mathrm{GmbH}$, Schwabmunchen, Germany), in which the abdomen was compressed under low pressure with foil. Thereafter, contrast-enhanced computed tomography (CT) scans were performed with a $2.5-\mathrm{mm}$ slice thickness on either a SOMATOM (Siemens, Berlin, Germany) or a LightSpeed RT16 (GE, Waukesha, WI, USA) CT scanner.

The gross tumor volume (GTV) was defined as the tumor volume that was enhanced in the arterial phase of the CT scan and diluted in the delayed phase. The planning target volume (PTV) was generated by the addition of 5-15 $\mathrm{mm}$ to the GTV according to the degree of respiratory movement. The organs at risk (OARs), such as the liver, stomach, duodenum, small and large intestines, both kidneys, and spinal cord, were also contoured.

The GTV was $127.4 \pm 158.5 \mathrm{~cm}^{3}$, and the PTV was $329.5 \pm 271.5 \mathrm{~cm}^{3}$. The total dose delivered to the PTV was 40-50 Gy (median, $50 \mathrm{~Gy}$ ) in 10 fractions. The dose was prescribed to $95 \%$ of the PTV. The normal tissue constraints were as follows: 1) total liver volume receiving at least $20 \mathrm{~Gy},<60 \%$; 2) mean liver dose, $<22 \mathrm{~Gy} ; 3$ ) mean kidney dose, $<13 \mathrm{~Gy}$; 4) $\mathrm{D}_{2 \mathrm{cc}}$ (the dose to $2 \mathrm{cc}$ volume of OARs) of the spinal cord, $<33 \mathrm{~Gy}$; and 4) $\mathrm{D}_{2 \mathrm{cc}}$ of the stomach, duodenum, and intestine, $<35 \mathrm{~Gy}$. We intended to perform the treatment plans based on the normal tissue constraints; however, these constraints were not always satisfied to achieve adequate target volume coverage. Treatment planning was performed by using the built-in software of the TomoTherapy Planning Station. We evaluated the dose-volume histogram and dose distributions in a slice-by-slice manner. We approved the treatment plan if the tumor coverage was adequate and the doses to the surrounding normal tissues were within clinically acceptable levels. Megavoltage cone-beam CT was performed during each treatment session before actual beam delivery. The patients' set-up was corrected by using automated image registration, and the anatomical accuracy was evaluated by a radiation oncologist.

\section{Evaluation and statistical analysis}

The effects of clinical factors, including age, sex, ECOG performance status, AJCC stage, presence or absence of liver cirrhosis, hepatitis, or PVTT, AFP level, pretreatment CP class, GTV, and prNLR, on the local progression-free survival (PFS) and overall survival (OS) were analyzed.

The OS duration was calculated from the date of RT to the date of death or the last follow-up date. The local PFS duration was calculated from the date of RT to the date of local disease progression, the last follow-up date, or the date of death. The local progression included the treated lesion only. The maximally selected log-rank test was used to determine the optimal cut-off value of
prNLR [29]. The parameters used in this analysis were 1) the presence or absence of local PFS, 2) local PFS duration, and 3) prNLR levels. The cumulative survival was calculated using the Kaplan-Meier method. Univariate and multivariate analyses were performed by using Cox proportional hazards models (Probability for stepwise: Entry-0.05, Removal-0.10, Maximum iterations-20, Method: Enter). Significant variables found in univariate analysis were included in multivariate analysis. The association between the clinical characteristics and the prNLR group was analyzed by using the chi-square test and independent $t$-test. Statistical analysis was performed with $\mathrm{R}$ version 3.1.2 (R Development Core Team, Vienna, Austria), and $p$ values $<0.05$ were considered statistically significant.

\section{CONFLICTS OF INTEREST}

The authors declare that they have no competing interests.

\section{REFERENCES}

1. Llovet JM, Bruix J. Systematic review of randomized trials for unresectable hepatocellular carcinoma: Chemoembolization improves survival. Hepatology. 2003; 37:429-442.

2. Lo CM, Ngan H, Tso WK, Liu CL, Lam CM, Poon RT, Fan ST, Wong J. Randomized controlled trial of transarterial lipiodol chemoembolization for unresectable hepatocellular carcinoma. Hepatology. 2002; 35:1164-1171.

3. Cheng AL, Guan Z, Chen Z, Tsao CJ, Qin S, Kim JS, Yang TS, Tak WY, Pan H, Yu S, Xu J, Fang F, Zou J, et al. Efficacy and safety of sorafenib in patients with advanced hepatocellular carcinoma according to baseline status: subset analyses of the phase III Sorafenib Asia-Pacific trial. Eur J Cancer. 2012; 48:1452-1465.

4. Llovet JM, Ricci S, Mazzaferro V, Hilgard P, Gane E, Blanc JF, de Oliveira AC, Santoro A, Raoul JL, Forner A, Schwartz M, Porta C, Zeuzem S, et al. Sorafenib in advanced hepatocellular carcinoma. N Engl J Med. 2008; 359:378-390.

5. Kim DY, Park W, Lim DH, Lee JH, Yoo BC, Paik SW, Kho KC, Kim TH, Ahn YC, Huh SJ. Three-dimensional conformal radiotherapy for portal vein thrombosis of hepatocellular carcinoma. Cancer. 2005; 103:2419-2426.

6. Yoon SM, Lim YS, Won HJ, Kim JH, Kim KM, Lee HC, Chung YH, Lee YS, Lee SG, Park JH, Suh DJ. Radiotherapy plus transarterial chemoembolization for hepatocellular carcinoma invading the portal vein: longterm patient outcomes. Int J Radiat Oncol Biol Phys. 2012; 82:2004-2011.

7. Son SH, Choi BO, Ryu MR, Kang YN, Jang JS, Bae SH, Yoon SK, Choi IB, Kang KM, Jang HS. Stereotactic body radiotherapy for patients with unresectable primary hepatocellular carcinoma: dose-volumetric parameters 
predicting the hepatic complication. Int J Radiat Oncol Biol Phys. 2010; 78:1073-1080.

8. Azab B, Bhatt VR, Phookan J, Murukutla S, Kohn N, Terjanian T, Widmann WD. Usefulness of the neutrophilto-lymphocyte ratio in predicting short- and long-term mortality in breast cancer patients. Ann Surg Oncol. 2012; 19:217-224.

9. Chua W, Charles KA, Baracos VE, Clarke SJ. Neutrophil/ lymphocyte ratio predicts chemotherapy outcomes in patients with advanced colorectal cancer. Br J Cancer. 2011; 104:1288-1295.

10. Fu SJ, Shen SL, Li SQ, Hua YP, Hu WJ, Liang LJ, Peng BG. Prognostic value of preoperative peripheral neutrophilto-lymphocyte ratio in patients with $\mathrm{HBV}$-associated hepatocellular carcinoma after radical hepatectomy. Med Oncol. 2013; 30:721.

11. Stotz M, Gerger A, Eisner F, Szkandera J, Loibner H, Ress AL, Kornprat P, AlZoughbi W, Seggewies FS, Lackner C, Stojakovic T, Samonigg H, Hoefler G, et al. Increased neutrophil-lymphocyte ratio is a poor prognostic factor in patients with primary operable and inoperable pancreatic cancer. Br J Cancer. 2013; 109:416-421.

12. Yamanaka T, Matsumoto S, Teramukai S, Ishiwata R, Nagai Y, Fukushima M. The baseline ratio of neutrophils to lymphocytes is associated with patient prognosis in advanced gastric cancer. Oncology. 2007; 73:215-220.

13. Yao Y, Yuan D, Liu H, Gu X, Song Y. Pretreatment neutrophil to lymphocyte ratio is associated with response to therapy and prognosis of advanced non-small cell lung cancer patients treated with first-line platinum-based chemotherapy. Cancer Immunol Immunother. 2013; 62:471-479.

14. Bertuzzo VR, Cescon M, Ravaioli M, Grazi GL, Ercolani G, Del Gaudio M, Cucchetti A, D’Errico-Grigioni A, Golfieri R, Pinna AD. Analysis of factors affecting recurrence of hepatocellular carcinoma after liver transplantation with a special focus on inflammation markers. Transplantation. 2011; 91:1279-1285.

15. da Fonseca LG, Barroso-Sousa R, Bento Ada S, Blanco BP, Valente GL, Pfiffer TE, Hoff PM, Sabbaga J. Pre-treatment neutrophil-to-lymphocyte ratio affects survival in patients with advanced hepatocellular carcinoma treated with sorafenib. Med Oncol. 2014; 31:264.

16. Dan J, Zhang Y, Peng Z, Huang J, Gao H, Xu L, Chen M. Postoperative neutrophil-to-lymphocyte ratio change predicts survival of patients with small hepatocellular carcinoma undergoing radiofrequency ablation. PLoS One. 2013; 8:e58184.

17. Liao W, Zhang J, Zhu Q, Qin L, Yao W, Lei B, Shi W, Yuan S, Tahir SA, Jin J, He S. Preoperative Neutrophilto-Lymphocyte Ratio as a New Prognostic Marker in Hepatocellular Carcinoma after Curative Resection. Transl Oncol. 2014; 7:248-255.
18. Tajiri K, Kawai K, Minemura M, Yasumura S, Hosokawa A, Kawabe H, Tomizawa G, Sugiyama T. Neutrophil/ lymphocyte ratio as a prognostic indicator of hepatic arterial infusion chemotherapy with arterial cisplatin plus continuous 5-fluorouracil. Hepatol Res. 2015; 45:755-763.

19. Terashima T, Yamashita T, Iida N, Yamashita T, Nakagawa H, Arai K, Kitamura K, Kagaya T, Sakai Y, Mizukoshi E, Honda M, Kaneko S. Blood neutrophil to lymphocyte ratio as a predictor in patients with advanced hepatocellular carcinoma treated with hepatic arterial infusion chemotherapy. Hepatol Res. 2014.

20. Wei K, Wang M, Zhang W, Mu H, Song TQ. Neutrophillymphocyte ratio as a predictor of outcomes for patients with hepatocellular carcinoma undergoing TAE combined with Sorafenib. Med Oncol. 2014; 31:969.

21. Kusumanto YH, Dam WA, Hospers GA, Meijer C, Mulder NH. Platelets and granulocytes, in particular the neutrophils, form important compartments for circulating vascular endothelial growth factor. Angiogenesis. 2003; 6:283-287.

22. Li X, Chen ZH, Ma XK, Chen J, Wu DH, Lin Q, Dong M, Wei L, Wang TT, Ruan DY, Lin ZX, Xing YF, Deng Y, et al. Neutrophil-to-lymphocyte ratio acts as a prognostic factor for patients with advanced hepatocellular carcinoma. Tumour Biol. 2014; 35:11057-11063.

23. Kuang DM, Zhao Q, Wu Y, Peng C, Wang J, Xu Z, Yin XY, Zheng L. Peritumoral neutrophils link inflammatory response to disease progression by fostering angiogenesis in hepatocellular carcinoma. J Hepatol. 2011; 54:948-955.

24. Kobayashi N, Hiraoka N, Yamagami W, Ojima H, Kanai Y, Kosuge T, Nakajima A, Hirohashi S. FOXP3+ regulatory $\mathrm{T}$ cells affect the development and progression of hepatocarcinogenesis. Clin Cancer Res. 2007; 13:902-911.

25. Pinato DJ, Stebbing J, Ishizuka M, Khan SA, Wasan HS, North BV, Kubota K, Sharma R. A novel and validated prognostic index in hepatocellular carcinoma: the inflammation based index (IBI). J Hepatol. 2012; 57:1013-1020.

26. Qi X, Li J, Deng H, Li H, Su C, Guo X. Neutrophilto-lymphocyte ratio for the prognostic assessment of hepatocellular carcinoma: A systematic review and metaanalysis of observational studies. Oncotarget. 2016; 7:45283-45301. doi: 10.18632/oncotarget.9942.

27. Grivennikov SI, Greten FR, Karin M. Immunity, inflammation, and cancer. Cell. 2010; 140:883-899.

28. Okamura Y, Sugiura T, Ito T, Yamamoto Y, Ashida R, Mori K, Uesaka K. Neutrophil to lymphocyte ratio as an indicator of the malignant behaviour of hepatocellular carcinoma. Br J Surg. 2016; 103:891-898.

29. Hothorn T, Zeileis A. Generalized maximally selected statistics. Biometrics. 2008; 64:1263-1269. 